\title{
Use of transcarotid artery revascularization for mechanical thrombectomy and treatment of symptomatic high-grade carotid artery stenosis associated with free-floating thrombus: illustrative case
}

\author{
Zachary K. Christian, MD, ${ }^{1}$ Alex N. Hoang, MD, ${ }^{2}$ Huy Dang, BA, ${ }^{3}$ Abdul B. Khan, MD, ${ }^{2}$ Daniel M. S. Raper, MBBS, ${ }^{2}$ \\ Zachary S. Pallister, $\mathrm{MD}^{4}$ and Omar Tanweer, $\mathrm{MD}^{2}$
}

Departments of ${ }^{1}$ General Surgery and ${ }^{2}$ Neurosurgery, and ${ }^{4}$ Division of Vascular Surgery and Endovascular Therapy, Baylor College of Medicine, Houston, Texas; and ${ }^{3}$ School of Medicine, Baylor College of Medicine, Houston, Texas

\begin{abstract}
BACKGROUND Patients with symptomatic high-grade stenosis of the internal carotid artery (ICA) associated with a free-floating thrombus (FFT) present a significant clinical challenge. In general, for patients with moderate to severe symptomatic ICA stenosis, carotid revascularization is recommended within 2 weeks of symptom onset; however, some physicians suggest that revascularization should be delayed in cases with FFT because some data suggest that early surgery with carotid endarterectomy or carotid stent poses a higher risk for stroke. Likewise, delayed revascularization with anticoagulation may increase risk of recurrent stroke. Few reports on the management of FTT included the use of a transcarotid artery revascularization (TCAR) approach for carotid revascularization with mechanical aspiration thrombectomy.
\end{abstract}

OBSERVATIONS This report described the use of TCAR for direct mechanical thrombectomy and carotid stent placement for a patient with $80 \%$ right ICA stenosis along with a large FFT extending into the bulb and the external carotid artery.

LESSONS The TCAR approach for mechanical thrombectomy and carotid stenting is a safe alternative for early revascularization with low periprocedural stroke risks.

https://thejns.org/doi/abs/10.3171/CASE21553

KEYWORDS TCAR; carotid artery stenosis; free-floating thrombus

The incidence of an intraluminal carotid thrombus increases with the degree of carotid stenosis present and ranges from $1.1 \%$ to a high of $5.5 \%$ in patients with $>85 \%$ stenosis. ${ }^{1}$ The presence of an intraluminal clot increases the 30-day risk of stroke or death, with double the risk when compared to surgically treated patients without a clot. ${ }^{1}$ The existence of an intraluminal clot poses a significant challenge mitigating periprocedural stroke risk and achieving good outcomes after surgery. This report describes the use of transcarotid artery revascularization (TCAR) for direct mechanical thrombectomy and carotid stent placement for a patient with $80 \%$ right internal carotid artery (ICA) stenosis along with a large free-floating thrombus (FFT) extending into the bulb and the external carotid artery.

\section{Illustrative Case}

A 62-year-old woman with a past medical history significant for left lower extremity deep vein thrombosis treated with an inferior vena cava filter, a family history of Factor $\mathrm{V}$ Leiden mutation, and a former tobacco smoking history (15 pack-years) presented with left arm weakness, slurred speech, and a facial droop. Computed tomography angiography of the carotid showed high-grade stenosis of the right cervical ICA with a nearly occlusive thrombus extending into the bulb and external carotid artery (Fig. 1). She was treated with anticoagulation with heparin infusion and antiplatelet therapy with aspirin $(81 \mathrm{mg})$ and clopidogrel $(75 \mathrm{mg})$. TCAR was performed after 5 days of dual antiplatelet therapy.

ABBREVIATIONS CAS = carotid artery stenting; $C C A=$ common carotid artery; CEA = carotid endarterectomy; FFT = free-floating thrombus; ICA = internal carotid artery; TCAR = transcarotid artery revascularization.

INCLUDE WHEN CITING Published March 7, 2022; DOI: 10.3171/CASE21553.

SUBMITTED September 28, 2021. ACCEPTED December 9, 2021.

(C) 2022 The authors, CC BY-NC-ND 4.0 (http://creativecommons.org/licenses/by-nc-nd/4.0/). 


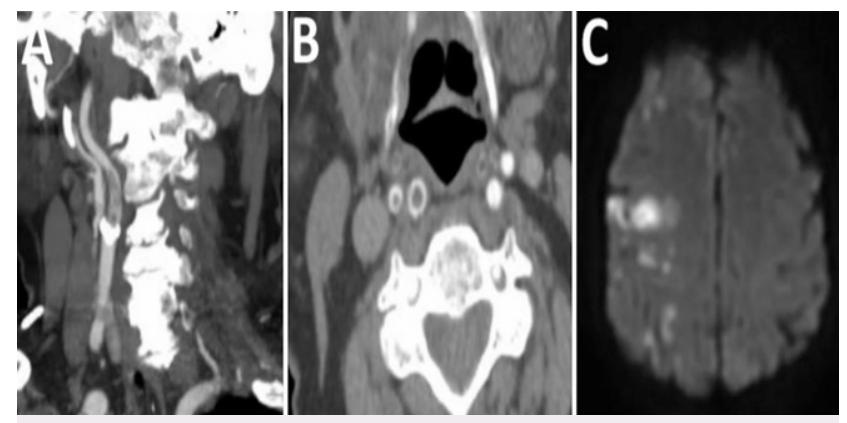

FIG. 1. Right cervical ICA with a nearly occlusive thrombus extending into the bulb and external carotid artery, seen on both sagittal $(\mathbf{A})$ and coronal (B) computed tomography angiography with the affected cerebral territories $(\mathbf{C})$.

A right TCAR approach with access to the right common carotid artery (CCA) and left common femoral vein was performed. After placement of the transcarotid arterial sheath in the CCA, the CCA inflow was occluded to achieve flow reversal with the external shunt and filter (flow controller). An AXS Catalyst 7 Distal Access Catheter (Stryker) with an outer diameter of 0.082 was inserted into the 8-Fr ENROUTE transcarotid arterial sheath under flow reversal. Aspiration thrombectomy, performed with repeat angiography under paused flow reversal, demonstrated resolution of most of the thrombus, with brisk flow into the intracranial arterial circulation. The ICA lesion was predilated with a 5-mm rapid exchange balloon. We then performed carotid stenting with an ENROUTE transcarotid stent $(9 \times 40 \mathrm{~mm})$. At TCAR case completion, antegrade flow was restored (Fig. 2). The patient tolerated the procedure well and was extubated on the table. The patient was moving all four extremities on command before transfer to the recovery room. On 30-day follow-up examination, her facial droop and slurred speech had resolved as well.

\section{Discussion}

\section{Observations}

Techniques used for the treatment of patients with ICA stenosis associated with an FFT included carotid endarterectomy (CEA), transfemoral carotid artery stenting (CAS), carotid sacrifice with vessel

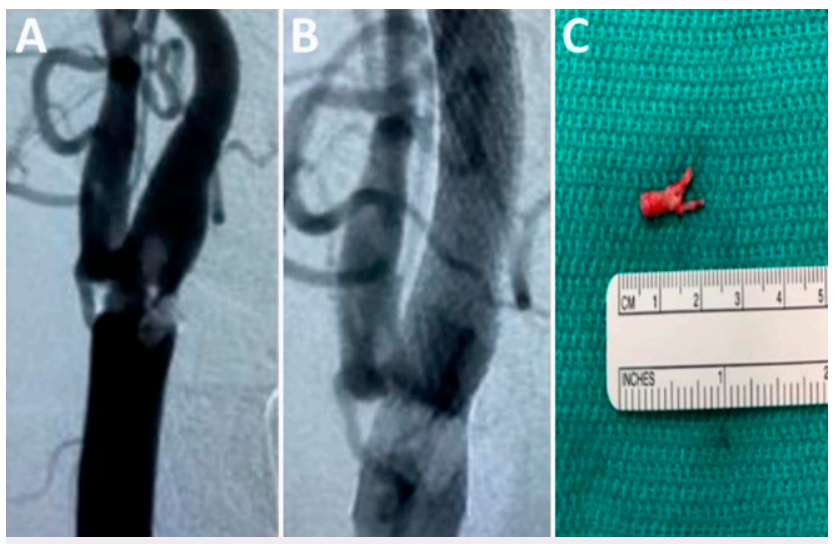

FIG. 2. Right internal common carotid arteriogram demonstrating an unstable FFT before (A) and after (B) aspiration thrombectomy and stenting. $\mathrm{C}$ : The fibrinous thrombus. embolization, and carotid artery bypass. ${ }^{2}$ Few reports have included mechanical aspiration thrombectomy ${ }^{3,4}$ or the use of a TCAR approach for carotid revascularization. ${ }^{5}$ Mareedu et al. showed a case of TCAR without mechanical thrombectomy for carotid stenosis associated with $\mathrm{FTT}^{5}$ Trapping of the FFT against the arterial wall with balloon and carotid stent is an option that enmeshes and secures the clot within the stent. This technique alone is not ideal for large clot burdens that fill the lumen because stenting the thrombus against the wall reduces the ICA luminal cross-section, contributing to stenosis. In addition, distal embolization can occur as the stent cells "slice" through the thrombus; therefore, this case required mechanical thrombectomy before stent placement.

Although use of TCAR in the setting of an FFT is technically outside the manufacturer's recommended indications, we took additional precautions during the procedure. First, we maintained flow reversal during the aspiration thrombectomy of the FFT in the cervical ICA. Second, during the thrombectomy, we remained ready to perform an intracranial thrombectomy in case of distal embolization. Third, we used a 0.072 aspiration catheter for suction aspiration.

CAS via a TCAR approach (Silk Road Medical, Inc.) has emerged as a minimally invasive and safe surgical approach for carotid revascularization. ${ }^{6}$ The TCAR technique requires direct common carotid access through a transcervical cutdown above the clavicle. Placing a large-bore sheath and obtaining proximal embolic protection through flow reversal with the ENROUTE transcarotid Neuroprotection System (NPS; Silk Road Medical, Inc.) allows for safe thrombectomy and traversing of the lesion for stent deployment. A systematic review of TCAR demonstrated that the 30-day neurological event rate for a TCAR procedure was $2 \%$ and the 30 -day death rate was $1 \%{ }^{6}$ In a systematic review and meta-analysis of TCAR outcomes among patients with symptomatic stenosis, the 30 -day mortality was $2.0 \%$, with the risk of stroke, transient ischemic attack, and cranial nerve injury being $1.6 \%, 0.8 \%$, and $0.36 \%$, respectively, ${ }^{7}$ values either comparable or superior to CEA and CAS. ${ }^{8-10}$

TCAR may be ideal for cases of carotid stenosis with an FFT in which CEA and transfemoral CAS may be a challenge. Carotid revascularization is recommended within 2 weeks of stroke index symptoms for patients with moderate to severe symptomatic ICA stenosis. In cases of FTT, early surgery in the presence of an unstable clot poses a higher risk for periprocedural stroke. ${ }^{2}$ The treatment paradigm includes anticoagulation or antiplatelet therapy with delayed carotid interventions, but there exists no validated study to direct treatment. CEA may be difficult because manipulation of the carotid bulb and proximal ICA may potentially dislodge the clot, leading to a major territory stroke; TCAR avoids forceful manipulation of the bifurcation and clot. Furthermore, the distal extension of the thrombus into the high cervical ICA would make cervical exposure difficult for CEA because of the inability to ensure ICA clamping distal to the thrombus to prevent clot migration. The TCAR approach with large-bore access of the CCA allows for direct safe thrombectomy with a 0.072 aspiration catheter for suction aspiration or stent retrieval in difficult cases.

\section{Lessons}

In conclusion, patients with symptomatic high-grade stenosis of the ICA associated with a thrombus present a significant clinical challenge. In addition, the standard of care in managing these patients has not been established, ${ }^{2}$ leaving it to clinicians to weigh the risks and benefits of available options in patient-specific settings to make 
the treatment decision with the best possible outcome. In general, for patients with moderate to severe symptomatic ICA stenosis, carotid revascularization is recommended within 2 weeks of symptom onset; however, some physicians suggest that revascularization should be delayed in cases with FFT because some data suggest that early surgery poses a higher risk of stroke., ${ }^{3,11}$ Unfortunately, the literature does not provide a comparison study that elucidates the true risk. Whether it is performed early or delayed, clinicians should consider TCAR to improve response rates in these patients because this procedure may decrease the risk of cranial nerve injury, operative time, and hospital length of stay in comparison to its counterparts. More data from robust, high-powered trials are needed to elucidate the true benefits of TCAR in treating symptomatic high-grade ICA stenosis with an FFT.

\section{References}

1. Barnett HJ. Efficacy of carotid endarterectomy translates to being efficacious with appropriate surgical skill. Arch Neurol. 2002; 59(12):1866-1868.

2. Fridman S, Lownie SP, Mandzia J. Diagnosis and management of carotid free-floating thrombus: a systematic literature review. Int $\mathrm{J}$ Stroke. 2019;14(3):247-256.

3. Buchan A, Gates P, Pelz D, Barnett HJ. Intraluminal thrombus in the cerebral circulation. Implications for surgical management. Stroke. 1988;19(6):681-687.

4. Park JW, Lee DH, Choi CG, Kim SJ, Suh DC. Various endovascular approaches to the management of free floating carotid thrombi: a technical report. J Neurointerv Surg. 2012;4(5):336-338.

5. Mareedu R, Hwang J, Vyakaranam S, Inkollu S. Use of transcarotid artery revascularization to treat symptomatic carotid artery stenosis associated with free-floating thrombus. Ann Vasc Surg. 2021;70: 568.e1-568.e3.

6. Nana PN, Brotis AG, Spanos KT, Kouvelos GN, Matsagkas MI, Giannoukas AD. A systematic review and meta-analysis of carotid artery stenting using the transcervical approach. Int Angiol. 2020;39(5):372-380.
7. Sagris M, Giannopoulos S, Giannopoulos S, et al. Transcervical carotid artery revascularization: a systematic review and metaanalysis of outcomes. J Vasc Surg. 2021;74(2):657-665.e12.

8. Murad MH, Shahrour A, Shah ND, Montori VM, Ricotta JJ. A systematic review and meta-analysis of randomized trials of carotid endarterectomy vs stenting. J Vasc Surg. 2011;53(3):792-797.

9. Plessers M, Van Herzeele I, Hemelsoet D, et al. Transcervical carotid stenting with dynamic flow reversal demonstrates embolization rates comparable to carotid endarterectomy. J Endovasc Ther. 2016;23(2):249-254.

10. Schermerhorn ML, Liang $P$, Dakour-Aridi $H$, et al. In-hospital outcomes of transcarotid artery revascularization and carotid endarterectomy in the Society for Vascular Surgery Vascular Quality Initiative. J Vasc Surg. 2020;71(1):87-95.

11. Vellimana AK, Kadkhodayan Y, Rich KM, et al. Symptomatic patients with intraluminal carotid artery thrombus: outcome with a strategy of initial anticoagulation. J Neurosurg. 2013;118(1):34-41.

\section{Disclosures}

The authors report no conflict of interest concerning the materials or methods used in this study or the findings specified in this paper.

\section{Author Contributions}

Conception and design: Christian, Pallister, Tanweer. Acquisition of data: Christian, Khan, Pallister, Tanweer. Analysis and interpretation of data: Christian, Hoang, Khan, Tanweer. Drafting the article: Christian, Hoang, Khan, Tanweer. Critically revising the article: Christian, Hoang, Raper, Pallister, Tanweer. Reviewed submitted version of manuscript: Christian, Hoang, Dang, Raper, Pallister, Tanweer. Approved the final version of the manuscript on behalf of all authors: Christian. Administrative/technical/material support: Christian, Dang, Pallister. Study supervision: Raper, Pallister.

\section{Correspondence}

Zachary K. Christian: Baylor College of Medicine, Houston, TX. zachary.christian@bcm.edu 